\title{
Stochastic processes and conformal invariance
}

\author{
Jan de Gier ${ }^{1}$, Bernard Nienhuis ${ }^{2}$, Paul A. Pearce ${ }^{1}$ and Vladimir Rittenberg ${ }^{1,3}$ \\ ${ }^{1}$ Department of Mathematics and Statistics, University of Melbourne, Parkville, Victoria 3010, Australia \\ ${ }^{2}$ Universiteit van Amsterdam, Valckenierstraat 65, 1018 XE Amsterdam, The Netherlands \\ ${ }^{3}$ Physikalisches Institut, Bonn University, 53115 Bonn, Germany.
}

(Dated: November 18, 2018)

\begin{abstract}
We discuss a one-dimensional model of a fluctuating interface with a dynamic exponent $z=1$. The events that occur are adsorption, which is local, and desorption which is nonlocal and may take place over regions of the order of the system size. In the thermodynamic limit, the time dependence of the system is given by characters of the $c=0$ logarithmic conformal field theory of percolation. This implies in a rigorous way a connection between logarithmic conformal field theory and stochastic processes. The finite-size scaling behavior of the average height, interface width and other observables are obtained. The avalanches produced during desorption are analyzed and we show that the probability distribution of the avalanche sizes obeys finite-size scaling with new critical exponents.
\end{abstract}

PACS numbers: 02.50.Ey, 11.25.Hf, 05.50.+q, 75.10.Hk

The structure of growing interfaces continues to be a subject of major interest and a characterization of the various universality classes of critical behavior remains an open question [1]. In this paper we present a onedimensional adsorption-desorption model of a fluctuating interface which belongs to a new universality class where the dynamical critical exponent $z=1$. In this model the interface evolves following nonlocal Markovian dynamics (for an adsorption-desorption model with local dynamics and $z>2$ see [2]). The relaxation rules are such that one has avalanches with a long tail in their probability distribution function (PDF). The model belongs therefore to the self-organized criticality class (SOC) [3, 1 , 5. Wh. What makes the model special is that the correlation lengths in the time and space directions are both proportional to the size of the system (this is not the case for other SOC models [4, 6]). The single scale that exists in the system is therefore its size. Moreover, our model has the big advantage of being solvable.

The Hamiltonian which gives the time evolution of our model is integrable. The finite-size scaling (FSS) limit of its spectrum can be obtained using the Bethe Ansatz [7] and is given by characters of a $c=0$ Virasoro algebra. A logarithmic conformal field theory (LCFT) with $c=0$ [8] appears also in other domains of physics such as systems with quenched disorder and the quantum Hall effect [9, and possibly string theory [10]. Moreover [11], the PDF describing the stationary state is related to combinatorial aspects of the ice model defined on a rectangle with special boundary conditions. This is probably the reason why we are able to conjecture exact expressions for various quantities connected to the model even for finite lattices.

We consider an interface on a one-dimensional lattice of size $L+1$ ( $L=2 n$ even). The non-negative heights $h_{i}(i=0,1, . ., L)$ that specify the interface, obey restricted solid-on-solid (RSOS) rules,

$$
h_{i+1}-h_{i}= \pm 1, \quad h_{0}=h_{L}=0, \quad h_{i} \geq 0
$$

Alternatively, we can describe the interface using slope variables $s_{i}=\left(h_{i+1}-h_{i-1}\right) / 2,\left(s_{0}=s_{L}=0\right)$. The interface evolves through adsorption and desorption according to the following rules. Adsorption, which locally changes the height $h_{i}$ to $h_{i}+2$, takes place with a rate equal to 1 at a local minimum of the interface $\left(s_{i}=0\right.$, $\left.h_{i}<\left(h_{i-1}, h_{i+1}\right)\right)$. The non-vanishing rates for desorption can be understood using the notion of active segments. A segment of a configuration with the end-points $a$ and $b$ is defined by the conditions: $h_{a}=h_{b}=h$ and $h_{i}>h$ for $a<i<b$. We call a segment active if at least one of the boundary slopes $s_{a}$ or $s_{b}$ is nonzero. In the desorption event, all the $b-a-1$ heights $h_{i}$ contained in a segment decrease by two units $\left(h_{i} \mapsto h_{i}-2\right)$ with a rate

$$
\delta\left(s_{a}-1\right)+\delta\left(s_{b}+1\right)
$$

where $\delta$ is the discrete Kronecker symbol, the other heights remaining unchanged. In order to find which desorption events can take place, for each of the $C_{n}=$ $(2 n) ! /\left((n+1)(n !)^{2}\right)$ configurations one first looks at how many active segments one has and then one uses (2). If the two slopes $s_{a}$ and $s_{b}$ are zero, the desorption rate is zero. This observation has two consequences. First, in the stationary state we expect to see predominantly configurations with large terraces, i.e. intervals where the slope is zero for all the sites. Next, it is meaningful to consider clusters. Those are segments where the endpoints have the heights equal to zero. According to the rules (2) desorption takes place only within a cluster.

The dynamics of the interface can easily be visualized using tiles (tilted squares) which cover the area between the interface and the substrate $\left(h_{2 i}=0, h_{2 i+1}=1\right)$. For a given configuration $c$, the number of tiles is $u(c)=$ $\frac{1}{2} \sum_{i=2}^{L-2} h_{i}+1-L / 4$. According to the rules given above, through adsorption one adds one tile, through desorption one loses a layer with $b-a-1$ tiles (this is an odd number). If one looks at our model from the point of view of self-organized critical phenomena, the number of tiles $u$ 
lost in a desorption event defines the size of an avalanche.

The physics of our model can be understood in the following way. We have a gas of tiles in the presence of a surface. Tiles uniformly try to attach to the interface and are succesful on sites that are lower than their neighbors. Tiles get reflected back into the gas on sites that are higher than their neighbors and trigger desorption events and are reflected on local slopes. Because the stationary state mainly consists of terraces, desorption events occur less frequently, but when they occur, they can take larger number of tiles.

Before describing the physical properties of the model we will give another formulation which enables us to obtain the FSS spectrum of the Hamiltonian, defined by the rates given above and thus to find the dynamic exponent. To each RSOS configuration with $L+1$ sites one can associate a configuration of $n$ non-intersecting half-loops on $L=2 n$ sites. The height $h_{i}$ is the number of half-loops above the midpoint of the sites $i$ and $i+1$ in the halfloop configuration. For example, the following picture illustrates the association between a configuration with three half-loops and an $L=6$ RSOS configuration with two clusters and one tile,

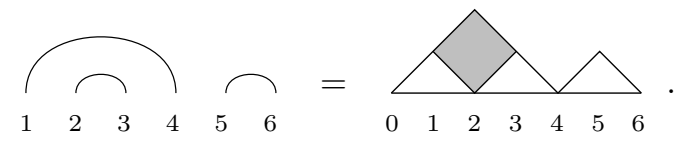

The half-loop configurations on the other hand, can be associated with the left ideal of the Temperley Lieb (TL) algebra at $Q=1$ [12]. The $L-1$ generators of the algebra satisfy the relations

$$
e_{i}^{2}=e_{i}, e_{i} e_{i \pm 1} e_{i}=e_{i},\left[e_{i}, e_{j}\right]=0,|i-j|>1,
$$

and have the graphical representation

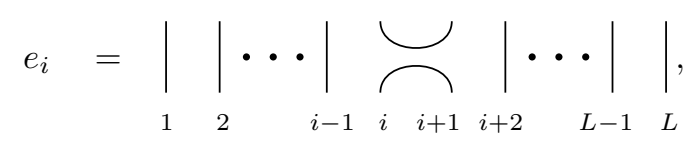

The left ideal is generated by the action of TL generators on $I_{0}=\prod_{i=1}^{n} e_{2 i-1}$. For example the action of $e_{3}$ on the half-loop configuration (3) is

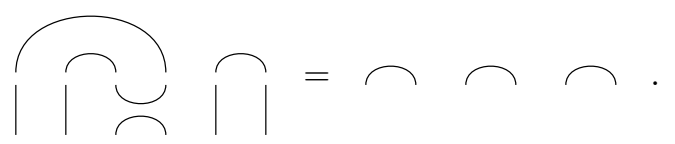

The Hamiltonian gives the time evolution in the vector space of the half-loop (RSOS) configurations,

$$
H=\sum_{j=1}^{L-1}\left(1-e_{j}\right), \quad \frac{\mathrm{d}}{\mathrm{d} t} P_{c}(t)=-\sum_{d} H_{c d} P_{d}(t) .
$$

$P_{c}(t)$ is the (unnormalized) probability to find the system in the configuration $c$ at the time $t$ and $r_{c d}=-H_{c d}$ give the rates of our model for the transitions $d \rightarrow c$. Since $H$ is an intensity matrix $\left(\sum_{c} H_{c d}=0\right)$ [11], it has a zero eigenvalue with a trivial bra and a nontrivial ket which gives the probabilities in the stationary state,

$$
\begin{aligned}
& \langle 0| H=0, \quad\langle 0|=(1,1, \ldots, 1), \\
& H|0\rangle=0, \quad|0\rangle=\sum_{c} P_{c}|c\rangle, \quad P_{c}=\lim _{t \rightarrow \infty} P_{c}(t) .
\end{aligned}
$$

Let us compute the dynamic critical exponent $z$. We use the following representation of the TL algebra

$$
\begin{aligned}
e_{i}= & \frac{1}{4}-\frac{1}{2}\left[\left(\sigma_{i}^{x} \sigma_{i+1}^{x}+\sigma_{i}^{y} \sigma_{i+1}^{y}-\Delta \sigma_{i}^{z} \sigma_{i+1}^{z}\right)\right. \\
& \left.+h\left(\sigma_{i}^{z}-\sigma_{i+1}^{z}\right)\right]
\end{aligned}
$$

where $\sigma^{x}, \sigma^{y}, \sigma^{z}$ are Pauli matrices, $-2 \Delta=q+q^{-1}=1$, $2 h=q-q^{-1}=1 \sqrt{3}$ and $q=\mathrm{e}^{\pi_{1} / 3}$. In this representation $H$ given by (7) becomes the Hamiltonian of the XXZ quantum chain 13] with $L$ sites and the energy gaps $E_{k}$ (the ground-state energy is zero for any $L$ ) scale like

$$
E_{k}=\pi v_{\mathrm{s}} x_{k} L^{-z},
$$

with $z=1, v_{\mathrm{s}}=3 \sqrt{3} / 2$ is the sound velocity [7] and $x_{k}$ are related to surface exponents of a $c=0$ LCFT of percolation 14]. In the continuum limit, the spectrum of the Hamiltonian (7), and thus the values of $x_{n}$, in the full TL algebra is given by the Virasoro characters [14]

$$
\chi_{s}(w)=w^{s(2 s-1) / 3}\left(1-w^{2 s+1}\right) \prod_{k=1}^{\infty}\left(1-w^{k}\right)^{-1} .
$$

Here $w=\exp \left(\pi T v_{\mathrm{s}} / L\right)$ parametrizes the temporal $(T)$ and spatial $(L)$ extent of the stochastic process. In the subspace of the RSOS configurations the spectrum is given by $\chi_{0}(w)$. To our knowledge, for the first time, a connection is made between stochastic processes and LCFT. This implies among other things that the space and time correlation lengths are the same and that, in the continuum limit, the forms of the space-time correlation functions in the stationary state are known [15].

As discussed in detail in 11 the stationary state of our model with $L$ sites is related to the two-dimensional ice model [16] defined on a rectangle of dimension $L \times$ $(L-1) / 2$ with special boundary conditions $[17,18]$. This model is equivalent to a fully packed loop (FPL) model 19] on the rectangle, all configurations being equally probable. We briefly explain this connection and its consequences. If we choose the (unnormalized) probability of the "pyramid" configuration $\left(s_{1}=\ldots=s_{n-1}=1\right.$, $s_{n}=0, L=2 n$ ) to be equal to one (this configuration has the smallest probability), then the normalization factor of the stationary state [20] is equal to

$$
\langle 0 \mid 0\rangle=\prod_{j=0}^{n-1}(3 j+2) \frac{(2 j+1) !(6 j+3) !}{(4 j+2) !(4 j+3) !}
$$

This is precisely $A_{\mathrm{V}}(2 n+1)$, the number of configurations of the FPL model on the rectangle. Moreover, the 
(unnormalized) probabilities for the other half-loop configurations are integer numbers which are equal to the numbers of configurations of the FPL model with the external occupied edges connected in the same way as in the configuration of half-loops [11, 21]. This implies that the PDF describing the stationary distribution in the RSOS configurations space corresponds to a uniform probability distribution in the space of the FPL model, or equivalently the ice model. The RSOS configuration with the largest (unnormalized) probability is the one with the longest terrace $\left(s_{1}=. .=s_{L}=0\right)$ in which the interface coincides with the substrate. The normalized PDF is obviously $p_{c}=P_{c} /\langle 0 \mid 0\rangle$.

We now describe some properties of the model. They were obtained either numerically for lattice sizes up to $L=18$ or an exact expression was conjectured and checked up to $L=18$. We start with the properties of the stationary state. The fraction of the interface covered by terraces 22] is

$$
\tau(L)=\frac{1}{L-1} \sum_{j=1}^{L-1}\left\langle 1-\left|s_{j}\right|\right\rangle=\frac{3 L^{2}-2 L+2}{(L-1)(4 L+2)} .
$$

This implies that for large $L$, three quarters of the surface is covered by terraces. We now consider the number of clusters per configuration $C=\sum_{j=1}^{L} \delta\left(h_{j}\right)$. Its average and the average of its square have the following expressions

$$
\begin{aligned}
\langle C\rangle & =\frac{1}{3} \prod_{j=0}^{n-1} \frac{(2 j+1)(3 j+4)}{(j+1)(6 j+1)}-\frac{1}{3} \approx 0.738 L^{2 / 3}, \\
\left\langle C^{2}\right\rangle & \approx\left(1.29-0.49 L^{-0.63}\right)\langle C\rangle^{2}
\end{aligned}
$$

From (15) which we deduce that the compressibility 23. of the gas of clusters $\kappa \approx 0.29 L$ diverges like the size of the system.

The average height $\langle\bar{h}\rangle$ and interface width $w_{\infty}$, which characterizes the roughness of the surface in the stationary state,

$$
\overline{h^{m}}=\frac{1}{L} \sum_{i=1}^{L} h_{i}^{m}, \quad w_{\infty}=\sqrt{\left.\overline{\left\langle h^{2}\right.}-\bar{h}^{2}\right\rangle}
$$

are compatible with the following behavior,

$$
\langle\bar{h}\rangle \approx 0.14 \ln (L / 2), \quad w_{\infty} \approx 0.34(\ln L / 2)^{1 / 3} .
$$

This implies that the PDF of the heights has a very small dispersion and also that the usual exponents [1] $\alpha=\beta$ vanish. As in any fit involving logs, the formulas given in (17) are probably not the last word. What is certain is that the width grows slowly with the size of the system implying that the surface is only marginally rough. It is interesting to mention that marginally rough surfaces (with $z=1.581$ corresponding to the directed percolation universality class) were also encountered 24 at a critical point dividing a moving rough KPZ phase from a smooth, massive phase. In these models a factor 3 between the powers of the logs (like in (17)) was also seen 25. The factor 3 is typical of a large class of growth problems [26]. To complete the interface growth picture, we calculated the ratio of the time dependent interface width $w(t, L)$ to $w_{\infty}$ for various lattice sizes. The curves were obtained by solving on a computer the differential equations (7) taking the substrate as the initial configuration. If $z=1$ and if the Family-Vicsek scaling applies, this ratio should converge to a function of $t / L$. We have checked that this is indeed the case.

We now consider the avalanches in our model. If in the stationary state one considers the configuration $d$ with $u(d)$ tiles, it changes with rate $r_{c d}$ into the configuration $c$ with $u(c)$ tiles. The rate of changes in which $u$ tiles are lost, can be written as

$$
R(u, L)=\sum_{c \neq d} \delta(u(d)-u(c)-u) r_{c d} p_{d}
$$

We have found the following values for the rate of adsorption of one tile, $R(u=-1, L)$, and the rate of all possible avalanches, $R(u>0, L)$,

$$
\begin{aligned}
R(-1, L) & =\frac{3 L(L-2)}{4(2 L+1)}, \\
R(u>0, L) & =L-2-2 R(-1, L) .
\end{aligned}
$$

Notice that for large $L$ the rate for the adsorption of one tile $(u=-1)$ is $3 / 2$ times larger than the rate for an avalanche $(u>0)$. This explains the relative rarity of the desorption events.

Since through desorption one loses an odd number of tiles, it is convenient to write $u=2 v-1$ and to consider $v$ as the size of an avalanche. Given the occurrence of an avalanche, its size $v$ is distributed according to the PDF

$$
S(v, L)=\frac{R(2 v-1, L)}{R(u>0, L)} .
$$

FSS [4] predicts the following form for this PDF,

$$
S(v, L)=v^{-\tau} F\left(v / L^{D}\right) .
$$

One way to get the exponents of the FSS function is to consider the moments [27,

$$
\left\langle v^{k}\right\rangle=\sum_{v=1} v^{k} S(v, L) \sim L^{\sigma(k)}
$$

and one expects

$$
\sigma(k)=\left\{\begin{array}{cc}
0, & k<\tau-1 \\
D(k+1-\tau), & k>\tau-1
\end{array} .\right.
$$

From equation (20) one gets $\langle v\rangle=(5 L+4) / 4(L+2)$, which implies that for large $L$ the average size of an avalanche is $3 / 2$ tiles. A numerical investigation of the other moments for $1 \leq k \leq 5$ indicates $\sigma(k)=k-2$. This implies $D=1$ and $\tau=3$. The numerics can be 


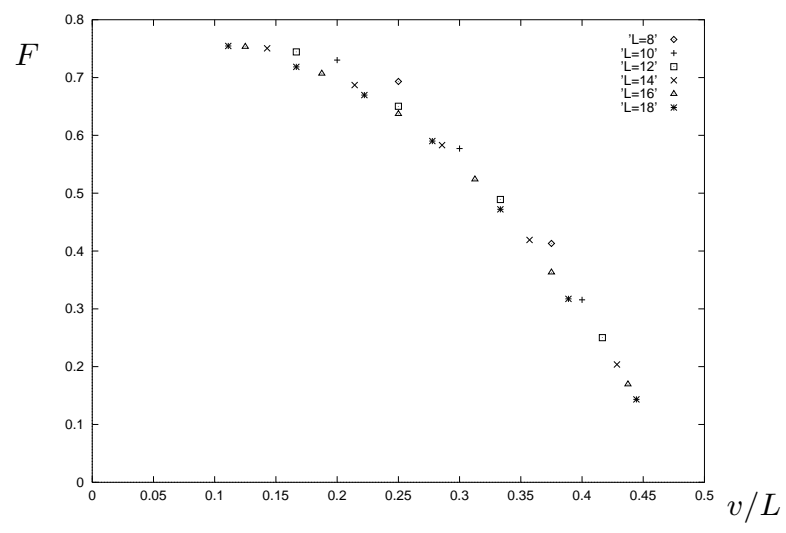

FIG. 1: Avalanche scaling function $F(v / L)$. The data are obtained for $v>1$ and $L=8, \ldots, 18$.

quite precise even if we have data for $L$ up to 18 . This is because we know $S(v, L)$ exactly and we can use VBS approximants [28] to derive the large $L$ behavior of the moments (the convergence is less good around $\sigma(k)=0$ due to logarithmic effects). The value $D=1$ was to be expected since $L$ is the only characteristic length in our system. A consistency check was done assuming $D=1$ in (22) to see for which value of tau one gets data collapse for the scaling function $F(v / L)$. Since we have data up to $L=18$ only we cannot expect a precise value of $\tau$ nor of $F$. Nevertheless as shown in Fig. 1, a data collapse is visible for $\tau=3.2$.
To conclude, we have presented an SOC model of a critical fluctuating interface belonging to a new universality class. We have also shown the connection between the model and a LCFT with $c=0$, which among other things implies that the dynamic exponent $z=1$. Several FSS exponents of expectation values have been computed in the stationary state but their identification with the scaling dimensions of the LCFT has not been completed. The FSS exponents of the avalanche PDF have also been determined. Much is still to be understood in this model, such as correlation functions and its off-critical behavior. We hope to come back to these topics in a future publication.

\section{Acknowledgements}

This research is supported by the Australian Research Council, and by the Foundation 'Fundamenteel Onderzoek der Materie' (FOM). JdG thanks the Australian National University where parts of this work were done. VR has been financially supported by ARC IREX and thanks the Einstein Centre of the Weizmann Institute and SISSA for hospitality. We thank M. T. Batchelor, E. Domany, M. Flohr, S. Franz, H. Hinrichsen, Y. Kafri, D. Mukamel, G. Mussardo, A. Nersesyan, A. Owczarek, Yu. Stroganov, A. Vespignani and O. Warnaar for discussions.
[1] Some recent reviews are A-L. Barabasi and H.E. Stanley, Fractal concepts in surface growth, Cambridge University Press, Cambridge (1995); E.H. Hinrichsen, Adv. Phys. 49, 815 (2000).

[2] H.M. Koduvely and D. Dhar, J. Stat. Phys. 90, 57 (1998).

[3] P. Bak, C. Tang and K. Wiesenfeld, Phys. Rev. Lett. 59, 381 (1987); J. Phys. A 38, 364 (1988).

[4] Some recent reviews are H. J. Jensen, Self Organised Criticality, Cambridge University Press, 1998; D. Dhar, Physica A 264, 1 (1999).

[5] A. Ben-Hur and O. Biham, Phys. Rev. E 53, R1317 (1996); A. Vespignani and S. Zapperi, Phys. Rev. E 57, 6345 (1998).

[6] S.V. Buldyrev, S. Havlin and H.E. Stanley, Physica A 200, 200 (1993);M. Paczuski, S. Maslov and P. Bak, Phys.Rev. E 53, 414 (1996).

[7] F. C. Alcaraz, M. N. Barber, M. T. Batchelor, R. J. Baxter and G. R. W. Quispel, J. Phys. A 20, 6397 (1987).

[8] I.I. Kogan and A. Nichols, arXiv:hep-th/0203207 and references therein.

[9] V.Gurarie and A.W.W. Ludwig, arXiv:condmat/9911392 I.I. Kogam and A.M. Tsvelik, Mod. Phys. Lett. A 15, 931 (2000).

[10] I.I. Kogan and D. Polyakov, Int. J. Mod. Phys. A 16, 2559 (2001).

[11] The mathematics related to the model is discussed in $P$. A. Pearce, V. Rittenberg, J. de Gier and B. Nienhuis, J.
Phys. A 35, L661 (2002).

[12] P.P. Martin, J.Phys. A 23, 7 (1990).

[13] V. Pasquier and H. Saleur, Nucl. Phys. B 330, 523 (1990).

[14] N. Read and H. Saleur, Nucl. Phys. B 613, 409 (2001); and references therein.

[15] C. Itzykson, H. Saleur and J-B. Zuber, Conformal invariance and applications to statistical mechanics, World Scientific Singapore 1988.

[16] E. Lieb, Phys. Rev. Lett. 18, 692 (1967).

[17] V. E. Korepin, Comm. Math. Phys. 86, 391 (1982);

[18] G. Kuperberg, math.CO/0008184 (2000);

[19] M. T. Batchelor, H. W. J. Blöte, B. Nienhuis and C. M. Yung, J. Phys. A 29, L399 (1996); B. Wieland Electron. J. Combin. 7, research paper 37 (2000).

[20] M. T. Batchelor, J. de Gier and B. Nienhuis, J. Phys. A 34, L265 (2001).

[21] A. V. Razumov and Yu. G. Stroganov, math.CO/0104216 (2001), math.CO/0108103 (2001);

[22] We thank Yu. G. Stroganov for this guess.

[23] R. K. Pathria, Statistical Mechanics, Pergamon Press, Oxford, 1972.

[24] U. Alon, M. R. Evans, H. Hinrichsen and D. Mukamel, Phys. Rev. E 57, 4997 (1998).

[25] H. Hinrichsen, private communication.

[26] P.J. Forrester, J. Phys. A 34, 417 (2001).

[27] C. Tebaldi, M. De Menech and A. L. Stella, Phys. Rev. 
Lett. 83, 3952 (1999).

10, 639 (1979).

[28] J.M. van den Broeck and L.W. Schwartz, J. Math. Anal. 